\title{
Oxygen Saturation-Dependent Absorption and Scattering of Blood
}

\author{
Dirk J. Faber, ${ }^{1}$ Maurice C. G. Aalders, ${ }^{1}$ Egbert G. Mik, ${ }^{2}$ Brett A. Hooper, ${ }^{3}$ \\ Martin J. C. van Gemert, ${ }^{1}$ and Ton G. van Leeuwen ${ }^{1,4}$ \\ ${ }^{1}$ Laser Center, Academic Medical Center, University of Amsterdam, Amsterdam, The Netherlands \\ ${ }^{2}$ Department of Physiology, Academic Medical Center, University of Amsterdam, Amsterdam, The Netherlands \\ ${ }^{3}$ Department of Biomedical Engineering and The Center for Emerging Cardiovascular Technologies, Duke University, \\ Durham, North Carolina 27708-0319, USA \\ ${ }^{4}$ Biophysical Engineering, Biomedical Technology Institute, University of Twente, Enschede, The Netherlands
} (Received 2 October 2003; published 8 July 2004)

\begin{abstract}
We report on the scattering properties of oxygenated and deoxygenated whole blood from 250 to $1000 \mathrm{~nm}$. We determine the complex refractive index of oxygenated and deoxygenated hemoglobin using a Kramers-Kronig analysis and optical coherence tomography measurements. Combining these data with Mie theory, the scattering properties are calculated. The strong oxygen saturation dependent scattering effects should be taken into account in the data analysis of optical oxymetry.
\end{abstract}

DOI: $10.1103 /$ PhysRevLett.93.028102

PACS numbers: 87.64.Cc, 42.25.Bs, 78.20.-e

Hemoglobin $(\mathrm{Hb})$, responsible for the transport of oxygen from the lungs to tissues, is primarily contained inside red blood cells (RBCs) and can reversibly bind up to four oxygen molecules to form oxygenated hemoglobin $\left(\mathrm{HbO}_{2}\right)$. The oxygen saturation $\left(\mathrm{SO}_{2}\right)$ is the statistical average of all oxygen bound to hemoglobin, relative to the total amount that can be bound. $\mathrm{SO}_{2}$ dependent absorption of light by blood has been studied extensively [1-4] because optical methods allow for noninvasive and continuous monitoring of saturation. Studies have focused on the wavelength range $250-1000 \mathrm{~nm}$. Here, absorption by blood is mainly determined by the concentration and $\mathrm{SO}_{2}$ level of hemoglobin since the absorption of water is low. Moreover, the $\mathrm{Hb}$ and $\mathrm{HbO}_{2}$ spectra exhibit characteristic features (Soret and $Q$ bands) in this wavelength range. Currently, the clinical use of optical methods to measure bulk tissue $\mathrm{SO}_{2}$ is widespread. These measurements are based on light extinction, which includes absorption and scattering losses along the path traveled by the light in the tissue. Scattering losses are not explicitly included in the data analysis, but are dealt with using calibration factors since the optical path length of the detected light is unknown and scattering properties vary from tissue type to tissue type and between individuals. Consequently, accurate quantitative $\mathrm{SO}_{2}$ measurements are not possible. Recently developed techniques based on low-coherence interferometry, e.g., optical coherence tomography (OCT) [5], allow for control over the optical path length traveled by the detected light and may thus resolve part of these problems. Moreover, it may enable highly localized $\mathrm{SO}_{2}$ measurements [6] of the microcirculation. To enable quantitative extinction based $\mathrm{SO}_{2}$ measurements, inclusion of scattering from RBCs in the data analysis is required. The main cause of scattering by blood is the refractive index mismatch between the hemoglobin solution inside the cell and its plasma environment. From the principle of causality and given the strong
$\mathrm{SO}_{2}$ dependent absorption spectra of hemoglobin, the refractive index of hemoglobin is also expected to be $\mathrm{SO}_{2}$ dependent and, consequently, so is scattering by RBCs. However, $\mathrm{SO}_{2}$ dependent whole blood scattering data are currently not available.

In this Letter, we present a novel method for retrieving the scattering properties of whole blood. From accurately known absorption spectra of $\mathrm{Hb}$ and $\mathrm{HbO}_{2}$ we derive their complex refractive index by the Kramers-Kronig (KK) relations in the wavelength range $250-1000 \mathrm{~nm}$ similar to the approach taken by Shumilina [7]. The obtained spectra are calibrated using OCT at $800 \mathrm{~nm}$ and are subsequently used to calculate the scattering properties of oxygenated and deoxygenated whole blood with Mie theory.

Knowledge of the complex refractive index $\mathbf{n}(\omega)=$ $n(\omega)+\mathbf{i} \kappa(\omega)$ of $\mathrm{Hb}$ and $\mathrm{HbO}_{2}$ is required to calculate the optical properties of oxygenated and deoxygenated blood. Here, $\kappa(\omega)$ relates to absorption coefficient $\mu_{a}$ of $\mathrm{Hb}$ or $\mathrm{HbO}_{2}$ through

$$
\kappa(\omega)=\frac{c \mu_{a}(\omega)}{2 \omega},
$$

where $c$ is the speed of light in vacuum and $\omega$ the angular frequency of the light. As for any causal system, $n(\omega)$ and $\kappa(\omega)$ are related through KK relations. For convenience we use a subtractive KK analysis [8], defined by

$$
\begin{aligned}
n(\omega)= & n\left(\omega_{0}\right) \\
& +\frac{2}{\pi}\left(\omega^{2}-\omega_{0}^{2}\right) P \int_{0}^{\infty} \frac{\omega^{\prime} \kappa\left(\omega^{\prime}\right)}{\left(\omega^{2}-\omega^{\prime 2}\right)\left(\omega_{0}^{2}-\omega^{\prime 2}\right)} d \omega^{\prime},
\end{aligned}
$$

where $n\left(\omega_{0}\right)$ is the refractive index measured at reference frequency $\omega_{0}$ to provide scaling of the calculated curves. In addition, this integral offers greater convergence than the conventional expression when data are available only 
on a limited frequency range. $P$ is the Cauchy principal value of the integral. Thus, knowledge of the absorption spectrum of the hemoglobin solution in combination with one measurement at a reference frequency allows determination of the refractive index spectrum of the hemoglobin solution.

To obtain reliable refractive index measurements it is vital to keep the hemoglobin samples in physiological conditions. OCT is used to measure $n\left(\omega_{0}\right)[9,10]$ of hemoglobin solutions in a closed circulation where $\mathrm{SO}_{2}$ can be precisely controlled. The OCT setup includes a Ti:sapphire laser (Femtolasers, Vienna), operating at center wavelength $800 \mathrm{~nm}\left(\omega_{0}=2.36 \times 10^{15} \mathrm{rad} / \mathrm{s}\right)$ with a $125 \mathrm{~nm}$ FWHM bandwidth. Dispersion in both interferometer arms is matched using a folded grating-lens pair in the reference arm. In-depth scanning is performed by moving the reference mirror mounted on a voice coil translator (V-102.2L, PI, $100 \mathrm{~nm}$ resolution), driven with a $1 \mathrm{~Hz}$ round-off triangular waveform. In the sample arm, the focusing lens $(f=25 \mathrm{~mm})$ is mounted on a similar but independently driven voice-coil translator to provide continuous matching of the position of the focus with the measurement position. The axial resolution is $3 \mu \mathrm{m}$. The interference signal $i(z)$ on the photodetector can be expressed as the convolution of the source autocorrelation $s_{i i}(z)$ and the sample's (complex) reflectance profile $R(z)$

$$
i(z)=s_{i i}(z) \otimes R(z)
$$

where $z$ is the position of the coherence gate inside the sample. The detector signal is bandpass filtered and coherently demodulated by a lock-in amplifier, digitized (8192 points per depth scan), and stored. The amplitude of $i(z)$ measured from a cuvette filled with a hemoglobin solution consists of two interference fringe envelopes corresponding to the glass-sample boundaries, separated by the cuvette's optical thickness OT $=n_{g r} d$. Here, $n_{g r}$ is the group refractive index of the hemoglobin solution and $d$ is the geometrical thickness of the cuvette lumen $(2.865 \mathrm{~mm})$. OT is determined by least-squares fitting $s_{i i}$ to the boundaries and calculating the separation of their centers; $n_{g r}$ is obtained from OT/d. The accuracy with which OT is determined depends on the width of $s_{i i}$ and its shape. Therefore a light source with broad, Gaussian shaped spectral bandwidth is required. At any frequency, $n_{g r}$ can be written as

$$
n_{g r}(\omega)=n(\omega)+\omega \frac{\partial n(\omega)}{\partial \omega},
$$

where $n(\omega)$ is the refractive index at $\omega$ as it appears in Eq. (2). The second right-hand side term of Eq. (4) can be obtained from Eq. (2) by differentiation to $\omega$; hence measurement of $n_{g r}(\omega)$ suffices to determine $n(\omega)$. The accuracy of this setup was tested using glucose-water solutions ranging from 1 to $10 \mathrm{~g} / \mathrm{l}$. Both the determined group refractive increment and the extrapolated group

028102-2 index of refraction of water were in excellent agreement with literature values [11].

Fresh porcine blood is anticoagulated before use. Nonscattering hemoglobin solutions are obtained by cell lysis and filtration as described before [6], resulting in a reduction of total hemoglobin concentration to $33 \%$, which is measured to be $93 \mathrm{~g} / 1$ using a Radiometer OSM3 blood gas analyzer. The solutions are maintained at $37^{\circ} \mathrm{C}$ and oxygen saturated at $0 \%$ and $100 \%$ using a Minimax plus hollow fiber clinical oxygenator (Medtronic). Before applying the subtractive KK analysis, the measured group indices of refraction are scaled to physiological hemoglobin concentration using

$$
n_{g r}(H b X)=n_{g r}\left(\mathrm{H}_{2} \mathrm{O}\right)+\Delta n_{g r, H b X}[H b X],
$$

where $n_{g r}\left(\mathrm{H}_{2} \mathrm{O}\right)$ is the group refractive index of water [1.3409 at $800 \mathrm{~nm}$, from Ref. [12] and using Eq. (4)], $\Delta n_{g r, H b X}$ the refractive increment and $[H b X]$ the hemoglobin species' concentration. The measured indices of refraction of $\mathrm{HbO}_{2}$ and $\mathrm{Hb}$ solutions at $800 \mathrm{~nm}$ are $1.392 \pm 0.001$ and $1.388 \pm 0.002$, respectively, average \pm SD of 1000 measurements. These values are in very close agreement with values reported in the literature, recalculated at $800 \mathrm{~nm}$ when appropriate [13-17]. In Ref. [7], using a similar KK analysis, but without experimental scaling, and in Ref. [18] higher values are reported. Deviations are most likely due to differences in hemoglobin concentration of the used samples and the fact that these experiments were performed on intact RBCs rather than hemoglobin solutions. Since these values are used as reference points for scaling the real refractive index [obtained by Eq. (2)], we verified that the means of the two data sets are significantly different using an unpaired $t$ test $(p<0.0001)$ [19]. The imaginary part of the $\mathbf{n}(\boldsymbol{\omega})$ is calculated using Eq. (1) with $\mu_{a}$ obtained from Ref. [4]. The KK analysis is performed by numerical evaluation of Eq. (2) over the frequency range $1.88 \times 10^{15}$ to $7.53 \times 10^{15} \mathrm{rad} / \mathrm{s}$ ( 250 to $1000 \mathrm{~nm}$ wavelength range). The resulting real part of the $\mathbf{n}(\boldsymbol{\omega})$ is shown in Fig. 1.

To calculate the scattering properties of whole blood, the RBC is assumed to be a homogeneous sphere as in most theoretical analyses on the interaction of light with blood. Scattering is then described by Mie theory, which has been shown to describe single scattering of light by RBCs well [20]. The basic results are the extinction and scattering cross sections $\left(\sigma_{e}\right.$ and $\left.\sigma_{s}\right)$ and the scattering anisotropy factor $g$ (their formulas can be found, e.g., in Ref. [21]) which are complex functions of the size parameter $\mathbf{x}$ and relative refractive index $\mathbf{m}$, given by

$$
\mathbf{x}=\frac{2 \pi a \mathbf{n}_{m}}{\lambda} \quad \text { and } \quad \mathbf{m}=\frac{\mathbf{n}_{s}}{\mathbf{n}_{m}}
$$

where $\mathbf{n}_{s}$ and $\mathbf{n}_{m}$ are the complex refractive index of the sphere and the medium, respectively, $a$ is the sphere radius, and $\lambda$ is the illuminating wavelength in vacuum. 


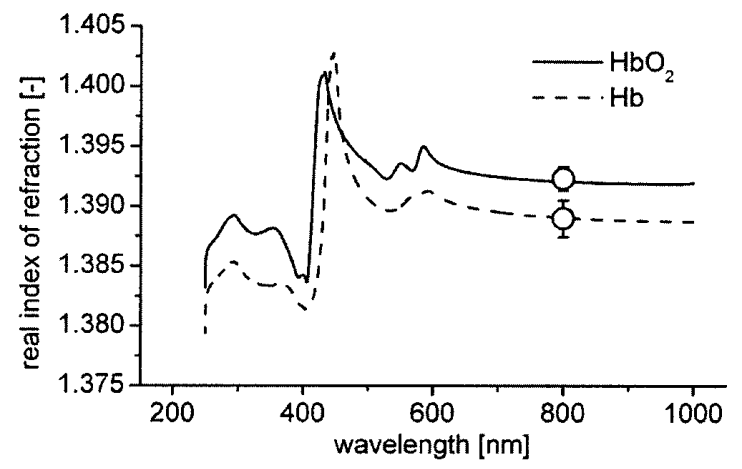

FIG. 1. Real part of the complex refractive index of $\mathrm{HbO}_{2}$ and $\mathrm{Hb}$ obtained from a subtractive $\mathrm{KK}$ analysis of the data of Ref. [4].

The cross sections $\sigma_{e, s}$ can be scaled to macroscopic coefficients using [21]

$$
\mu_{e, s}=\frac{H}{V} \sigma_{e, s} .
$$

Here, $H$ is the particle volume fraction (for blood called hematocrit), and $V$ is the sphere volume. The $\mu_{e}$ accounts for both scattering and absorption losses. The $\mu_{a}$ is obtained by subtracting $\mu_{s}$ from $\mu_{e}$. For the Mie calculations the code by Zijp et al. [22] was modified to take complex arguments. In Eq. (6) we use $\mathbf{n}_{m}=1.340$ [23] as the refractive index of plasma at $800 \mathrm{~nm}$, and $a=$ $2.78 \mu \mathrm{m}$, corresponding to a typical $\mathrm{RBC}$ volume of $V=$ $90 \mu \mathrm{m}^{3}$. In Eq. (7) $H=0.5$ is taken. Figure 2 shows the calculated scattering coefficient and Fig. 3 the scattering anisotropy vs wavelength of oxygenated and deoxygenated whole blood. In the wavelength range $400-500 \mathrm{~nm}$, corresponding to the location of large absorption peaks in the $\mathrm{Hb} / \mathrm{HbO}_{2}$ absorption spectra, large scattering changes are observed varying from 200 to $425 \mathrm{~mm}^{-1}$. For $\lambda>600 \mathrm{~nm}$ scattering by oxygenated blood is about $10 \%$ higher than scattering by deoxygenated blood; in this wavelength range $\mu_{s}$ decreases with increasing wavelength. The $g$ is slightly lower for oxygenated blood for $\lambda>600 \mathrm{~nm}$. The effect of the uncertainty in the

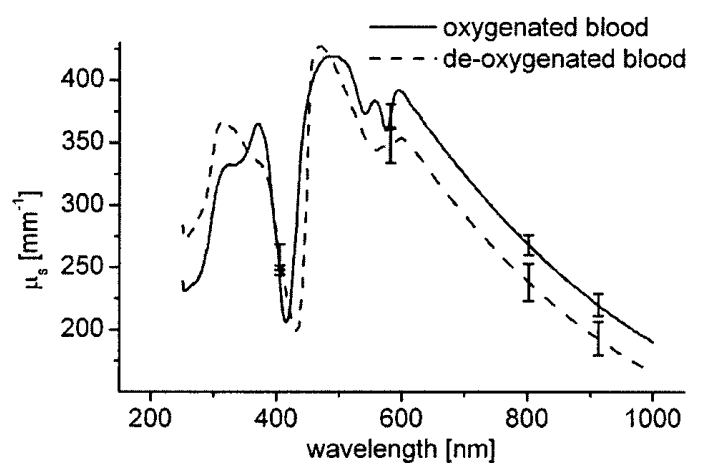

FIG. 2. Scattering coefficient vs wavelength of oxygenated and deoxygenated whole blood computed by Mie theory using the calculated complex refractive indices.

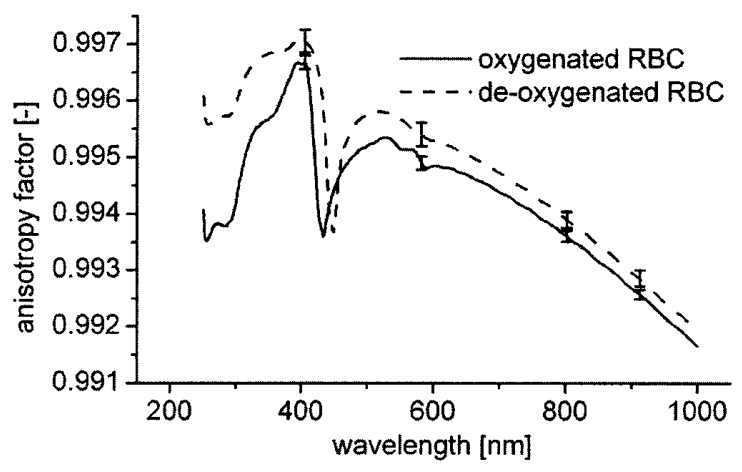

FIG. 3. Anisotropy factor vs wavelength of an oxygenated and deoxygenated RBC computed by Mie theory using the calculated complex refractive indices.

reference measurement on the scattering properties was assessed by repeating the Mie calculations with $n(\omega)$ now scaled by $n\left(\omega_{0}\right) \pm \mathrm{SD}$. The deviation at $800 \mathrm{~nm}$ and at the clinically relevant wavelengths 405,580 , and $910 \mathrm{~nm}$ is depicted by error bars in Figs. 2 and 3. For the whole spectral range, deviations in $\mu_{s}$ are less than $4 \%$ (8\%) for oxygenated (deoxygenated) whole blood; deviations in $g$ are less than $1 \%$. Consistency of the method was tested by calculating $\mu_{a}=\mu_{e}-\mu_{s}$. Good agreement with the absorption data of Ref. [4] (Fig. 4) is found. Some care should be taken with regard to the absolute values of the $\mu_{s}$. The scaling with $H$ assumes independent scattering. With $H=0.5$ this assumption is not obvious. Alternative scaling factors have been proposed in literature [24]. These, however, do not change the shape of the presented curves, only their amplitude. For low $H$, our calculated data show good agreement with the experimental optical data of oxygenated diluted blood by Roggan et al. measured using a double integrating sphere technique [25]. However, contrary to our results, variations of scattering coefficient and anisotropy with $\mathrm{SO}_{2}$ did not reach significance. Measurements of $\mu_{s}$ of oxygenated whole blood using the same technique by

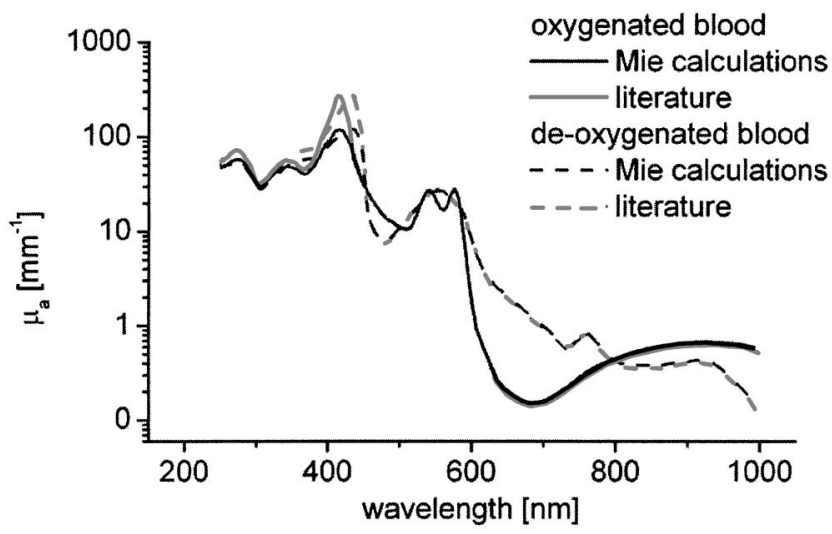

FIG. 4. Absorption coefficient vs wavelength of oxygenated and deoxygenated whole blood. Gray curves: data from Ref. [4]. Black curves: Mie calculations. 
Yaroslavsky et al. [26] showed qualitative agreement with our data in the wavelength range 700-1000 $\mathrm{nm}$. However, an increase in $g$ with wavelength was found. The highly forward scattering of blood, with $g$ reported between 0.985 and 0.997 [23,23-27], makes it difficult to separate scattered from nonscattered light, e.g., required in integrating sphere measurements, implying that the optical properties of whole blood are difficult to determine experimentally. Moreover, aggregation and biochemical responses, such as clotting, make it especially challenging to perform measurements of ex vivo whole blood samples under controlled physiological conditions. Extraction of optical properties from such measurements is complex, and usually requires an inverse Monte Carlo technique $[25,26]$. Consequently, a wide variety of experimental values have been reported [16,23,25-27]. The approach outlined in this Letter avoids the necessity of measurements on whole blood and using complex reconstruction algorithms. Rather, we determine the refractive index of nonscattering hemoglobin solutions using the KramersKronig relations, and calculate the scattering properties of oxygenated and deoxygenated whole blood. The choice of Mie theory to perform the latter calculations is not essential. Other, possibly more accurate scattering theories (e.g., Ref. [17,23]) also take the complex refractive index as input, and it is its $\mathrm{SO}_{2}$ dependence that determines the $\mathrm{SO}_{2}$ dependent scattering properties. Our method therefore provides a template for accurate evaluation of the blood scattering properties.

To summarize, in this Letter we present experimental values for the refractive index of oxygenated and deoxygenated hemoglobin at $800 \mathrm{~nm}$. The former is significantly larger than the latter, which has not been identified before. The scattering properties of oxygenated and deoxygenated whole blood are determined by a physically straightforward novel method based on accurately known hemoglobin absorption spectra and Kramers-Kronig relations to obtain the complex refractive index as input for Mie theory. This methodology is particularly advantageous for retrieving scattering properties of whole blood since it does not require keeping whole blood under controlled physiological conditions, uses nonscattering hemoglobin solutions instead of highly (forward) scattering blood samples, and does not require complex inverse strategies. It is shown that not only the absorption coefficient but also the scattering properties strongly depend on $\mathrm{SO}_{2}$. Therefore, to enable quantitative (regional) tissue $\mathrm{SO}_{2}$ measurements, it is vital to include both in data analysis.

This work is part of the research program of the "Stichting voor Fundamenteel Onderzoek der Materie (FOM)," which is financially supported by the "Nederlandse Organisatie voor Wetenschappelijk
Onderzoek (NWO)." NWO supported part of the program. We thank Alexei Kharine for translating Ref. [7] from Russian.

[1] O.W. van Assendelft, Spectrophotometry of Heamoglobin Derivatives (Royal Vangorcum Ltd., Assen, 1970).

[2] W. G. Zijlstra, A. Buursma, and W. P. Meeuwsen-van der Roest, Clin. Chem. 37, 1633 (1991).

[3] S. Wray et al., Biochim. Biophys. Acta 933, 184 (1988).

[4] Tabulated data from various sources compiled by S. Prahl at http://omlc.ogi.edu/spectra.

[5] D. Huang et al., Science 254, 1178 (1991).

[6] D. J. Faber et al., Opt. Lett. 28, 1436 (2003).

[7] F. S. Shumilina, Akademiya Navuk Belarusskai SSSR Vestsi-Serrya Fizika-Matematychnykh Navuk 1, 79 (1984).

[8] R. K. Ahrenkiel, J. Opt. Soc. Am. 61, 1651 (1971).

[9] A. Knuettel and M. Boehlau-Godau, J Biomed. Opt. 5, 83 (2000).

[10] X. Wang et al., J. Biomed. Opt. 7, 628 (2002).

[11] Measured $n_{g r}=1.343 \pm 0.001 ; n_{g r}=1.3409$ [12] and using Eq. (4). Measured $\Delta n_{g r}=(1.8 \pm 0.2) \times 10^{4} \mathrm{l} / \mathrm{g}$; $\Delta n_{g r}=(1.67 \pm 0.01) \times 10^{4} \mathrm{l} / \mathrm{g}$ at $850 \mathrm{~mm} ; \mathrm{J}$.S. Maier et al., Opt. Lett. 19, 2062 (1994); G. S. Nikolic, M. D. Cakic, and L. A. Ilic, J. Serb Chem. Soc. 66, 397 (2001).

[12] H. D. Downing and D. Williams, J. Geophys. Res. 80, 1656 (1975).

[13] W. H. Orttung and J. Warner, J. Phys. Chem. 69, 3188 (1965).

[14] J. L. Stoddard and G. S. Adair, J. Chem. Biol. 57, 437 (1923).

[15] R. A. MacRae, J. A. McClure, and P. Latimer, J. Opt. Soc. Am. 51, 1366 (1961).

[16] L. R. Reynolds, C. Johnson, and A. Ishimaru, Appl. Opt. 15, 2059 (1976).

[17] M. Hammer et al., Appl. Opt. 37, 7410 (1998).

[18] A. Ya. Khairullina, Opt. Spektrosc. (USSR) 53, 623 (1982).

[19] Douglas G. Altman, Practical Statistics for Medical Research (Chapman \& Hall, London, 1997).

[20] J. M. Steinke and A. P. Shepherd, Appl. Opt. 27, 4027 (1988).

[21] H. C. van de Hulst, Light Scattering by Small Particles (Dover Publications, Inc., New York, 1957).

[22] J. R. Zijp and J. J. ten Bosch, Opt. Eng. 32, 1691 (1993).

[23] Handbook of Biomedical Optics, edited by V.V. Tuchin (SPIE Press, Bellingham, 2002).

[24] V. Twersky, J. Opt. Soc. Am. 60, 1084 (1970).

[25] A. Roggan et al., J. Biomed. Opt. 4, 36 (1999).

[26] A. N. Yaroslavsky et al., Proc. SPIE Int. Soc. Opt. Eng. 2678, 314 (1996).

[27] W. F. Cheong, S. A. Prahl, and A. J. Welch, IEEE J. Quantum Electron. 26, 2166 (1990). 